\title{
Transmission of light through periodic arrays of square holes: From a metallic wire mesh to an array of tiny holes
}

\author{
J. Bravo-Abad, ${ }^{1}$ L. Martín-Moreno, ${ }^{2}$ F. J. García-Vidal,,${ }^{1} *$ E. Hendry, ${ }^{3}$ and J. Gómez Rivas ${ }^{4}$ \\ ${ }^{1}$ Departamento de Física Teorica de la Materia Condensada, Universidad Autonoma de Madrid, E-28049 Madrid, Spain \\ ${ }^{2}$ Departamento de Fisica de la Materia Condensada, ICMA, Universidad de Zaragoza, E-50009 Zaragoza, Spain \\ ${ }^{3}$ Thin Films Photonics Group, School of Physics, University of Exeter, Stocker Road, Exeter EX4 4QL, United Kingdom \\ ${ }^{4}$ FOM Institute for Atomic and Molecular Physics AMOLF, clo Philips Research Laboratories, High Tech Campus 4, \\ 5656 AE Eindhoven, The Netherlands
}

(Received 30 October 2007; published 7 December 2007)

\begin{abstract}
A complete landscape is presented of the electromagnetic coupling between square holes forming a twodimensional periodic array in a metallic film. By combining both experimental and theoretical results along with a first-principles Fano model, we study the crossover between the physics of metallic wire meshes (when holes occupy most of the unit cell) and the phenomenon of extraordinary optical transmission, which appears when the size of the holes is very small in comparison with the period of the array.
\end{abstract}

DOI: $10.1103 /$ PhysRevB.76.241102

Metallic wire meshes have been studied since the middle of the last century, due to their use as wavelength-selective filters. Their bandpass behavior is based on the appearance of a transmission peak located at a wavelength close to the period of the array. As the area occupied by the square holes is very large, the peak transmission wavelength is shorter than the cutoff wavelength of the hole waveguide, $\lambda_{C}$. Then, electromagnetic (EM) fields inside the holes have a propagating character. These structures have been extensively analyzed at microwave, ${ }^{1}$ far-infrared, ${ }^{2}$ mid-infrared, ${ }^{3}$ and infrared ${ }^{4}$ frequencies.

On the other hand, the discovery of the phenomenon of extraordinary optical transmission (EOT) through twodimensional (2D) periodic arrays of subwavelength holes in optically thick metal films ${ }^{5}$ has renewed the interest in analyzing how light interacts with subwavelength apertures. ${ }^{6-11}$ In such hole arrays, resonant wavelengths $\left(\lambda_{M}\right)$ are spectrally located near the period of the array. For small holes, $\lambda_{M}>\lambda_{C}$ and the EM fields inside the holes present an evanescent decay. As a difference with metallic wire meshes in which the film thickness is much smaller than $\lambda_{M}$, in the EOT regime metal thicknesses are typically of the order of one-half of the period of the array. In Ref. 5, resonant transmission peaks were recorded in the optical range of the EM spectrum. Subsequently, EOT has also been observed at terahertz $(\mathrm{THz})$ and microwave frequencies. ${ }^{12-15}$

The fundamental understanding of these two different regimes is the following. Resonant features appearing for small holes result from the coupling, via the periodicity of the array, of the incident light to surface EM modes. ${ }^{6}$ In essence, the nature of these surface EM modes depends on the frequency regime we are considering. At optical frequencies, they are surface plasmons, ${ }^{8}$ while at $\mathrm{THz}$ or microwave frequencies (where metals behave as perfect conductors), these surface EM modes have a geometric origin and result from the collective EM interaction between the holes. ${ }^{16}$ In contrast, when holes occupy most of the unit cell in a metallic wire mesh, the transmission peak is explained ${ }^{17}$ in terms of the singularity of the scattered EM field that appears when the first diffractive orders emerge from the array at a grazing angle (at the so-called Rayleigh wavelength $\lambda_{R}$ ). At this particular wavelength, the weak EM coupling between holes is increased, resulting in a peak in the transmittance spectrum. Note that these two limiting cases (surface EM mode and Rayleigh singularity) correspond to the two general types of Wood's anomalies that are present in optical gratings, as discussed in Ref. 18.

The aim of this Rapid Communication is to study the crossover between these two distinct regimes by analyzing the evolution of the transmission spectra of 2D arrays of square holes as a function of the size of the holes. In order to do that, we combine experimental and theoretical results with an $a b$ initio Fano model, able to capture the physics of the transition. Our study will be carried out in an intermediate scenario in which the metal film is extremely thin and is deposited on top of a high-index dielectric substrate (silicon).

From the experimental side, we have considered four perforated gold films in which both the film thickness $(h=0.51 \mu \mathrm{m})$ and the period of the array $(d=100 \mu \mathrm{m})$ are fixed. The sides of the holes in the four samples are $a=68$, 58,48 , and $38 \mu \mathrm{m}$. The metallic films are deposited on top of a Si substrate $(\epsilon=11.7)$. THz transmission was measured using a pulsed $\mathrm{THz}$ time-domain spectrometer based on an InGaAs emitter and a photoconductive antenna detector similar to that described in Ref. 19. The pulses transmitted through the samples are Fourier transformed to obtain the transmittance, defined as the transmission power normalized by the transmission through a bare Si substrate.

Figure 1(a) shows the experimental transmission spectra obtained for the four samples. For $a=68 \mu \mathrm{m}$, the spectrum is dominated by a peak appearing at $\lambda_{M}=350 \mu \mathrm{m}$, very close to the Rayleigh wavelength $\lambda_{R}=d \sqrt{\epsilon}=342 \mu \mathrm{m}$. This peak is the canonical transmission feature observed in metallic wire meshes. ${ }^{3}$ As the side of the square holes is decreased, the height of the transmission peak is strongly reduced and its location shifts slightly to longer wavelengths. Together with this reduction and shift of the resonant peak, a transmission minimum located at a shorter wavelength than the corresponding peak appears in the spectrum. The emergence of a transmission dip is also observed close to the second-order 

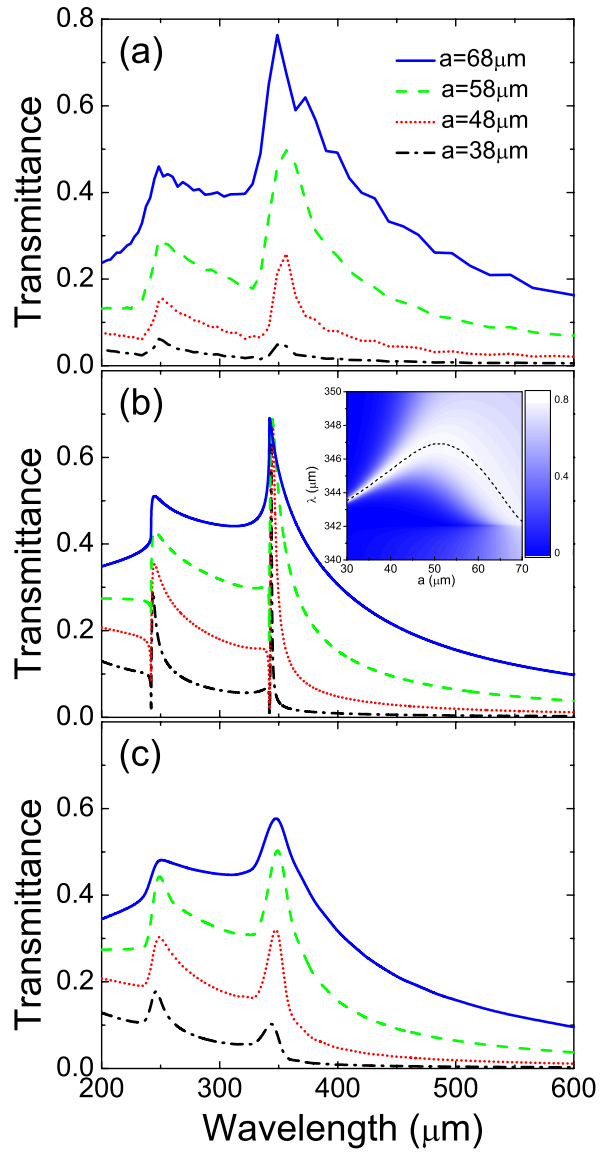

FIG. 1. (Color online) (a) Measured zero-order transmittance spectra corresponding to the four samples. The spectra were obtained under normal incident radiation. (b) Calculated zero-order transmittance for an infinite periodic array of holes with the same geometrical parameters as in (a). Inset shows the transmittance as a function of wavelength and side of the holes $(a)$, which is varied continuously from $a=30$ to $70 \mu \mathrm{m}$. The dot-dash line displays the spectral location of the transmission peak for each value of $a$. (c) Calculated transmittance for a $21 \times 21$ hole array.

resonance located at $240 \mu \mathrm{m}$. This type of behavior (transmission peaks with adjacent dips located at shorter wavelengths) is a general property of the light transmission through periodic arrays of subwavelength apertures, as it also appears in 1D arrays of slits. ${ }^{20,21}$

In order to understand this evolution of the transmission spectra, we have performed numerical simulations of the structures. We use a theoretical framework based on the modal expansion of the EM fields in the different regions of the system. This formalism treats the metallic regions within the perfect conductor approximation, i.e., we assume $\epsilon=-\infty$ inside the metal, which is an excellent approximation for gold at $\mathrm{THz}$ frequencies. Within this framework, EM fields in the reflection and transmission regions are expanded in terms of $s$ - and $p$-polarized plane waves whereas inside the holes, $\mathbf{E}$ and $\mathbf{H}$ fields are written as a linear combination of the TE and TM waveguide modes. By matching the parallel components of the EM fields at the two interfaces of the structure, a system of linear equations for the expansion coefficients of the EM fields inside the holes can be built up. It is convenient to express the expansion coefficients in terms of the $E$-field mode amplitudes at the illuminated $\left(E_{\alpha}\right.$, where $\alpha$ denotes a particular waveguide mode) and nonilluminated $\left(E_{\alpha}^{\prime}\right)$ surfaces of the structure. More details can be found in Ref. 22. Once the set $\left[E_{\alpha}, E_{\alpha}^{\prime}\right]$ is known (by solving the set of linear equations), EM fields in all space can be extracted and the zero-order transmittance through the structure calculated as

$$
T=\sum_{\alpha}\left|E_{\alpha}^{\prime}\right|^{2}\left|S_{\alpha}\right|^{2}
$$

where $S_{\alpha}$ is the overlapping integral between the zero-order plane wave and mode $\alpha$ inside the hole.

Figure 1(b) depicts the calculated zero-order transmittance spectra for infinite arrays of holes corresponding to the four values of $a$ analyzed in the experiments (transmission is normalized to the energy flux impinging at the unit cell of the structure). The evolution of the transmission spectra is better captured in the inset of Fig. 1(b) in which a contour plot of the transmittance is displayed as a function of the wavelength and the size of the holes. For $a=68 \mu \mathrm{m}$, a transmission peak emerges at $\lambda_{R}$ in good agreement with the experiments. As the size of the holes is reduced, two main changes take place in the spectra. The location of the transmission peak first moves to longer wavelengths, but this trend changes for $a \approx 50 \mu \mathrm{m}$ (see the dot-dash line in the inset). For $a<50 \mu \mathrm{m}$, the transmission peak shifts toward $\lambda_{R}$. Additionally, as $a$ is reduced, a dip emerges at $\lambda_{R}$ and the transmittance is virtually zero at the dip for $a<50 \mu \mathrm{m}$. As a technical note, a well-converged result for the transmittance is obtained, for all the cases analyzed here, by considering only one channel when evaluating $T$ from Eq. (1). The dominant contribution comes from the mode $\mathrm{TE}_{01}$, the least evanescent mode inside the holes (from now on we label this mode as $\alpha=0)$. However, an accurate evaluation of $E_{0}^{\prime}$ to feed Eq. (1) makes it necessary to take into account several waveguide modes in addition to the $\mathrm{TE}_{01}$ mode.

In contrast to the experimental results in Fig. 1(a), the heights of the transmission peaks in Fig. 1(b) are almost the same in the four structures analyzed. Also the dip is much more pronounced in the theoretical spectra than in the experimental ones. By using the theoretical formalism described above, it is also possible to calculate the transmission properties of finite collections of holes. ${ }^{22}$ This is done in Fig. 1 (c) for a $21 \times 21$ array of holes with the same geometrical parameters as in Fig. 1(b). The agreement between theory and experiment is much better, indicating that the discrepancies between Figs. 1(a) and 1(b) arise from the inherent finite-size effects of the experimental setup. ${ }^{22,23}$

From now on we concentrate on understanding the physical origin of the transition under study. In order to do that, it is convenient to take advantage of the fact that the transmittance is governed by mode $\mathrm{TE}_{01}$. Then, we can write $E_{0}^{\prime}$ as $E_{0}^{\prime}=E_{0}^{s h}\left(1+\Delta E_{0}\right), E_{0}^{s h}$ being the zero-mode amplitude of the electric field at the nonilluminated side for a single, isolated hole and $\Delta E_{0}$ the new contribution to this amplitude when the hole is placed in a $2 \mathrm{D}$ array. This decomposition allows us to separate explicitly the resonant and nonresonant parts of $E_{0}^{\prime}$. As a consequence, Eq. (1) can be expressed as 

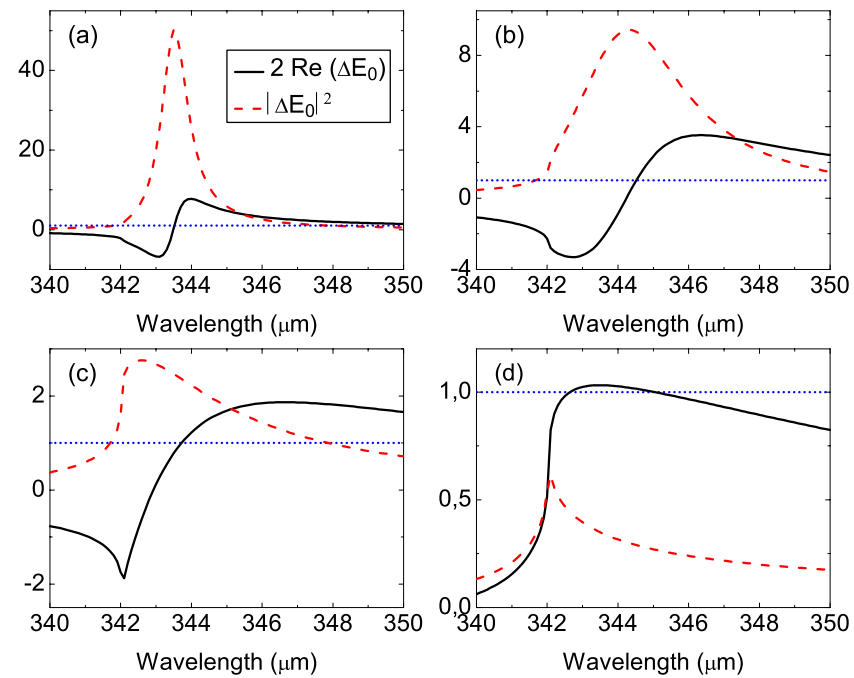

FIG. 2. (Color online) Plots of the three different terms contributing to the transmission through the hole array as written in Eq. (2) for $a=$ (a) 38, (b) 48, (c) 58, and (d) $68 \mu \mathrm{m}$. The rest of the geometrical parameters are as in Fig. 1. The red (gray) dashed lines and solid black lines correspond to the resonant $\left(\left|\Delta E_{0}\right|^{2}\right)$ and interference $\left[2 \operatorname{Re}\left(\Delta E_{0}\right)\right]$ terms, respectively. These magnitudes are normalized to the nonresonant contribution [dotted blue (dark gray) lines, constant value 1].

$$
T=\left|S_{0}\right|^{2}\left|E_{s h}\right|^{2}\left(1+\left|\Delta E_{0}\right|^{2}+2 \operatorname{Re}\left[\Delta E_{0}\right]\right) .
$$

It is important to note that this expression closely resembles a Fano-type formula in which two different mechanisms (resonant and nonresonant channels) interplay in the final transmission process. ${ }^{24}$ The first term in Eq. (2) is the contribution to the transmittance of each hole as if they were in an array where there was no EM interaction between them. This term can be identified as the nonresonant channel in the Fano picture. The second term corresponds to the resonant contribution coming from the EM coupling between the holes in a 2D array. Finally, the third term gives the interference between the resonant and nonresonant channels. We emphasize that, in contrast with other Fano approaches to EOT, ${ }^{25}$ our model does not contain any fitting parameter (i.e., it is an ab initio Fano model for EOT).

The dependence of the contributions to the transmittance given by Eq. (2) on the size of the holes is analyzed in Fig. 2. As depicted in Fig. 2(a), for the smallest holes $(a=38 \mu \mathrm{m})$, the resonant contribution [red (gray) dashed line] is much larger that the nonresonant one [blue (dark gray) dotted line], i.e., $\left|\Delta E_{0}\right|^{2} \gg 1$. This contribution can be interpreted as the one coming from the excitation of surface EM modes at the metallic interfaces. ${ }^{6,16}$ As in a typical resonant phenomenon, the interference term (solid black line) changes its sign very rapidly, resulting in the appearance of a minimum and a maximum in the transmittance at locations very close to the resonant condition [see Fig. 1(b)]. In this case the minimum can reach a zero value as the condition $\operatorname{Re}\left(\Delta E_{0}\right)=-\left(1+\left|\Delta E_{0}\right|^{2}\right) / 2 \approx-\left|\Delta E_{0}\right|^{2} / 2$ can be satisfied.

As the value of $a$ is increased [see Fig. 2(b), $a=48 \mu \mathrm{m}$ ] the resonant term broadens and its peak shifts to longer wavelengths, these two effects being related to the EM cou-

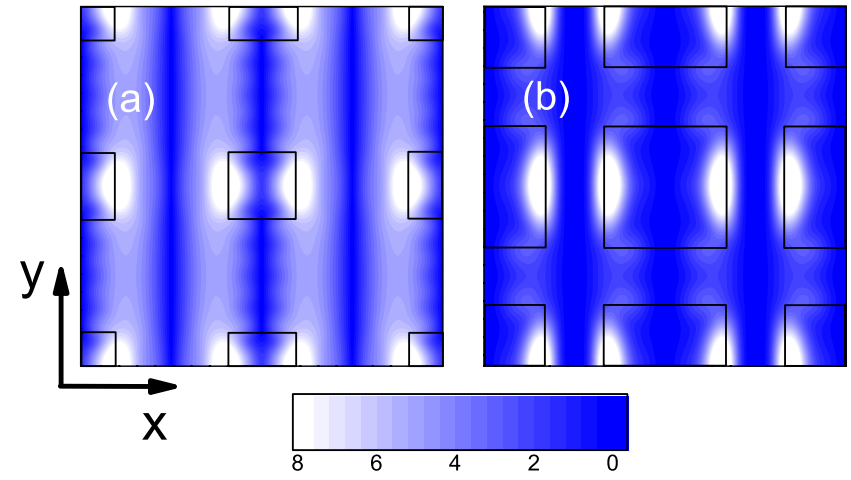

FIG. 3. (Color online) Electric field patterns at resonance corresponding to $a=$ (a) 38 and (b) $68 \mu \mathrm{m}$. The rest of the geometrical parameters are as in Figs. 1 and 2. Both patterns were calculated just above the nonilluminated side of the samples and the amplitude is normalized to the incident one. Black square boxes in both panels correspond to the perimeters of the holes in the two cases.

pling of the surface mode with radiative modes. Also, its magnitude is decreased with respect to the single-hole contribution. However, the interference term still cancels exactly the sum of the resonant and single-hole contributions [see Fig. 1(b)]. This situation changes when $a$ is larger than half of the period $(50 \mu \mathrm{m})$. This change of behavior is illustrated in Fig. 2(c), which shows the $a=58 \mu \mathrm{m}$ case. Now the peak of the resonant term has moved to shorter wavelengths (very close to $\lambda_{R}$ ) and its height is strongly reduced. The interference term now presents a sharp minimum that induces a nonzero dip in the transmission spectrum [see Fig. 1(b)]. When $a$ is further increased [see Fig. 2(d), $a=68 \mu \mathrm{m}$ ], the term associated with the EM coupling between holes is even smaller than the single-hole contribution and the interference term is always positive in the range of interest. Although smaller, the contribution coming from the EM interaction between holes is peaked exactly at the Rayleigh wavelength $\lambda_{R}$, reflecting the fact that, in this regime, the coupling between holes is maximum when a diffraction order glances the surface. Note that, as the scattered EM field is singular at $\lambda_{R}$, the derivative of this contribution is discontinuous at the peak wavelength. Additionally, in this limit there is no transmission dip as the interference term presents no minimum.

In addition to the Fano model, we have also developed an iterative way of solving the system of equations for $\left[E_{\alpha}, E_{\alpha}^{\prime}\right]$. In this procedure the EM interaction between holes is introduced by means of a perturbation series. The transmission peak at the Rayleigh wavelength in the case of weaker EM coupling (large holes) can be obtained after a few iterative steps, while a very large number of iterations are needed when the resonance is due to the presence of a surface EM mode (actually, the iteration may even fail to converge for wavelengths very close to those of the surface mode).

In order to visualize the transition in the physics of the transmission peak when the size of the holes is increased, we have computed the electric field amplitude at the resonant wavelength for $a=38$ and $68 \mu \mathrm{m}$ just above the nonilluminated side of the samples [Figs. 3(a) and 3(b), respectively]. For $a=38 \mu \mathrm{m}$ we observe two fingerprints of the surface EM 
mode formed by the holes: (i) the electric field is mainly concentrated in the metallic regions and (ii) a wave front appears in the $y$ direction, indicating the propagation of the surface EM mode in the $x$ direction (as was expected, as the incident electric field was chosen to point along the $x$ axis). At normal incidence, since scattering to surface EM modes with wave vectors in the $x$ direction is isotropic, this wave front defines a standing wave. In contrast, for $a=68 \mu \mathrm{m}$, these two features disappear: the electric field is concentrated inside the holes and the wave front is not present, showing that the EM interaction between holes is very small.

In conclusion, we have analyzed in detail the evolution of the transmission properties of a $2 \mathrm{D}$ array of square holes when the size of the holes is varied. We have shown that there is a continuous transition going from a resonant transmission regime governed by the excitation of surface electromagnetic modes when the area of the holes is small to a scenario in which the EM coupling between holes is weak and mediated by grazing diffraction orders when they occupy most of the unit cell. Our results provide the missing link relating the physics of metallic wire meshes to the phenomenon of extraordinary optical transmission.

This work has been funded by the Spanish MEC under Contract No. MAT2005-06608-C02. It was also supported by the Netherlands Foundation "Fundamenteel Onderzoek der Materie (FOM)" and the "Nederlandse Organisatie voor Wetenschappelijk Onderzoek (NWO)."
*Corresponding author; fj.garcia@uam.es

${ }^{1}$ F. Keilmann, Int. J. Infrared Millim. Waves 2, 1259 (1981).

${ }^{2}$ A. Mitsuishi et al., Jpn. J. Appl. Phys. 2, 574 (1963).

${ }^{3}$ R. Ulrich, Infrared Phys. 7, 37 (1967).

${ }^{4}$ C. M. Rhoads, E. K. Damon, and B. A. Munk, Appl. Opt. 21, 2814 (1982).

${ }^{5}$ T. W. Ebbesen, H. J. Lezec, H. Ghaemi, T. Thio, and P. A. Wolff, Nature (London) 391, 667 (1998).

${ }^{6}$ L. Martín-Moreno, F. J. García-Vidal, H. J. Lezec, K. M. Pellerin, T. Thio, J. B. Pendry, and T. W. Ebbesen, Phys. Rev. Lett. 86, 1114 (2001).

${ }^{7}$ R. Gordon, A. G. Brolo, A. McKinnon, A. Rajora, B. Leathem, and K. L. Kavanagh, Phys. Rev. Lett. 92, 037401 (2004).

${ }^{8}$ W. L. Barnes, W. A. Murray, J. Dintinger, E. Devaux, and T. W. Ebbesen, Phys. Rev. Lett. 92, 107401 (2004).

${ }^{9}$ K. J. Klein Koerkamp, S. Enoch, F. B. Segerink, N. F. van Hulst, and L. Kuipers, Phys. Rev. Lett. 92, 183901 (2004).

${ }^{10}$ A. P. Hibbins, M. J. Lockyear, I. R. Hooper, and J. R. Sambles, Phys. Rev. Lett. 96, 073904 (2006).

${ }^{11}$ F. J. García de Abajo, J. J. Saenz, I. Campillo, and J. S. Dolado, Opt. Express 14, 7 (2006).

${ }^{12}$ J. Gómez Rivas, C. Schotsch, P. Haring Bolivar, and H. Kurz, Phys. Rev. B 68, 201306(R) (2003).

${ }^{13}$ H. Cao and A. Nahata, Opt. Express 12, 1004 (2004).
${ }^{14}$ D. Qu, D. Grischkowsky, and W. Zhang, Opt. Lett. 29, 896 (2004).

${ }^{15}$ M. Beruete, M. Sorolla, I. Campillo, J. S. Dolado, L. MartínMoreno, J. Bravo-Abad, and F. J. García-Vidal, Opt. Lett. 29, 2500 (2004).

${ }^{16}$ J. B. Pendry, L. Martín-Moreno, and F. J. García-Vidal, Science 305, 847 (2004).

${ }^{17}$ N. Amitay, V. Galindo, and C. P. Wu, Theory and Analysis of Phased Array Antennas (Wiley, New York, 1972).

${ }^{18}$ A. Hessel and A. A. Oliner, Appl. Opt. 10, 1275 (1965).

${ }^{19}$ M. C. Beard, G. M. Turner, and C. A. Schmuttenmaer, J. Phys. Chem. B 106, 7146 (2002).

${ }^{20}$ U. Schröter and D. Heitmann, Phys. Rev. B 60, 4992 (1999).

${ }^{21}$ J. A. Porto, F. J. García-Vidal, and J. B. Pendry, Phys. Rev. Lett. 83, 2845 (1999).

22 J. Bravo-Abad, F. J. García-Vidal, and L. Martín-Moreno, Phys. Rev. Lett. 93, 227401 (2004).

${ }^{23}$ J. Bravo-Abad, A. Degiron, F. Przybilla, C. Genet, F. J. GarcíaVidal, L. Martín-Moreno, and T. W. Ebbesen, Nat. Phys. 2, 120 (2006).

${ }^{24}$ U. Fano, Phys. Rev. 124, 1866 (1961).

${ }^{25}$ C. Genet, M. P. van Exter, and J. P. Woerdman, Opt. Commun. 225, 331 (2003). 